\title{
Comparison of Fixed Burst Versus Decremental Burst Pacing for Termination of Ventricular Tachycardia
}

\author{
HUGH CALKINS, RAFEL EL-ATASSI, STEVE KALBFLEISCH, \\ JONATHAN LANGBERG, and FRED MORADY \\ From the University of Michigan Medical Center Division of Cardiology, Ann Arbor, Michigan
}

\begin{abstract}
CALKINS, H., ET AL:: Comparison of Fixed Burst Versus Decremental Burst Pacing for Termination of Ventricular Tachycardia. The objective of this study was to compare prospectively the efficacy of fixed burst pacing with that of decremental burst pacing in terminating VT. Forty-four patients with inducible sustained monomorphic VT were studied. The efficacy of fixed burst and decremental burst pacing for terminating 57 distinct types of VT were compared during 50 electrophysiology tests (mean VT cycle length $=334 \pm 84 \mathrm{msec}$ ). Termination of each type of VT was attempted with fixed burst and decremental burst pacing. Both pacing algorithms were delivered in an adaptive fashion with an increasing number of stimuli with each successive attempt at VT termination. Seventy percent of VT episodes were successfully terminated with fixed burst pacing. The mean number of stimuli required for VT termination was $5 \pm 2$. Seventy-two percent of VT episodes were successfully terminated with decremental burst pacing. The mean number of stimuli required for VT termination was $5 \pm 2$. For fixed burst and decremental burst pacing, the efficacy of VT termination was greater for VTs with a cycle length $>300$ msec than for faster VTs $(P<0.05)$. The efficacy of VT termination and the incidence of VT acceleration were no different for the two pacing algorithms $(P>0.1)$. The results of this study demonstrate that fixed burst and decremental burst pacing are equally effective in terminating VT and that a single adaptive pacing algorithm is effective in terminating nearly three fourths of VTs. (PACE, Vol. 16, January, Part I 1993)
\end{abstract}

decremental pacing, burst pacing, ventricular tachycardia, antitachycardia pacing

\section{Introduction}

Recently, implantable cardioverter defibrillators have been developed that are capable of antitachycardia pacing as well as low and high energy cardioversion/defibrillation. ${ }^{1-3}$ Although these devices can be programmed to deliver a wide range of pacing schemes, the optimal antitachycardia pacing algorithm remains uncertain. Only one study to date has compared the efficacy of decremental burst pacing and fixed burst pacing for termination of ventricular tachycardia (VT). ${ }^{4} \mathrm{Al}-$

Address for reprints: Hugh Calkins, M.D., Johns Hopkins Hospital, Electrophysiology, Carneige, Room 530, 601 Wolfe Street, Baltimore, MD 21287. Fax: (410) 614-1345.

Received February 20, 1992; revision April 29, 1992; accepted May 21, 1992. though decremental burst pacing was found to be more effective, the two pacing algorithms differed significantly in the number of impulses that were delivered, the coupling intervals used, and the method of delivery. Therefore, it is unclear whether fixed burst or decremental burst pacing is more effective.

The objective of this study was to compare prospectively the efficacy of fixed burst pacing with that of decremental burst pacing for terminating VT.

\section{Methods}

\section{Patient Population}

The subjects of this study were 44 consecutive patients who had reproducibly inducible sustained monomorphic VT during electrophysiolog- 
ical testing and who agreed to participate in the study. The presenting arrhythmia was sustained monomorphic VT in 37 patients, ventricular fibrillation in five patients, and syncope in two patients. There were 39 men and five women with a mean age of $65 \pm 9$ years ( 1 standard deviation). Thirty-seven patients had a history of coronary artery disease and myocardial infarction, four patients had an idiopathic dilated cardiomyopathy, two patients had hypertensive heart disease, and one patient had no structural heart disease. The mean left ventricular ejection fraction was $0.34 \pm$ 0.17 .

\section{Electrophysiological Testing}

Electrophysiological studies were performed in the fasting state. The study was approved by the Human Research Committee at the University of Michigan and informed consent was obtained in each patient. Quadripolar electrode catheters were inserted into a femoral vein and positioned at the high right atrium, across the tricuspid valve to record a His-bundle electrogram, and at the apex of the right ventricle. Leads V1, I and III, and the intracardiac electrograms were recorded at a paper speed of $25 \mathrm{~mm} / \mathrm{sec}$ using a Siemens-Elema Mingograph 7 recorder (Siemens-Elema, Solna, Sweden). Programmed stimulation was performed with a programmable stimulator (Bloom Associates Ltd., Reading, PA, USA) with stimuli that were $2 \mathrm{msec}$ in duration and had a current intensity of twice diastolic threshold.

Programmed ventricular stimulation was performed with up to three extrastimuli using 8-beat drive cycle lengths of 600,400 , and $350 \mathrm{msec}$. If sustained monomorphic VT was not induced at the apex, programmed stimulation was repeated at the right ventricular outflow tract.

\section{Evaluation of Antitachycardia Pacing Algorithms}

The efficacy of fixed burst and decremental burst pacing in terminating 57 distinct types of VT were compared during 50 electrophysiological studies. Thirty-three patients had the efficacy of antitachycardia pacing evaluated only during antiarrhythmic drug therapy, six patients had the efficacy of antitachycardia pacing evaluated only in the absence of antiarrhythmic drugs, and five pa- tients had the efficacy of antitachycardia pacing evaluated in the baseline state and during antiarrhythmic drug therapy. The mean VT cycle length was $334 \pm 84 \mathrm{msec}$ (range $230-560 \mathrm{msec}$ ). Twenty-five VTs had a left bundle configuration; 14 with a superior axis and 11 with an inferior axis. Thirty-two VTs had a right bundle configuration; 19 with a superior axis and 13 with an inferior axis.

Termination of each type of VT was attempted with fixed burst and decremental burst pacing. The order in which each pacing scheme (fixed burst versus decremental burst) was delivered was randomized. Pacing therapy was delivered at the apex of the right ventricle using a stimulus strength of $7.5 \mathrm{~V}$ and a pulse width of $2 \mathrm{msec}$. The pacing algorithms were delivered through a standard quadripolar electrode catheter positioned at the apex of the right ventricle using a custom-designed automated multiprogrammable antitachycardia pacemaker. The pacing threshold was $<1 \mathrm{~mA}$ in all cases.

For each attempt at termination of VT, the pacing algorithm was classified as being successful, unsuccessful, or resulting in acceleration of the VT. Acceleration of VT was considered to have occurred if there was $>$ a $25 \%$ decrease in the tachycardia cycle length or if polymorphic VT or ventricular fibrillation were induced. The number of attempts of the pacing algorithm required for termination of VT was also recorded.

\section{Antitachycardia Pacing Algorithms}

The two antitachycardia pacing algorithms that were tested in this study are described schematically in Figure 1. During fixed burst pacing, a train of impulses was delivered during VT with a coupling interval of $91 \%$ of the VT cycle length. The coupling interval of the second stimulus was $81 \%$ of the VT cycle length. The initial number of pulses delivered was four. During each successive attempt, the number of pacing impulses were increased by one and the pacing cycle length was shortened by 16 msec. A maximum of five attempts with a maximum of eight impulses were delivered. Decremental burst pacing was delivered using a similar protocol. The train was again coupled to the VT with a coupling interval of $91 \%$ and the second stimulus of the train was delivered 
CALKINS, ET AL.
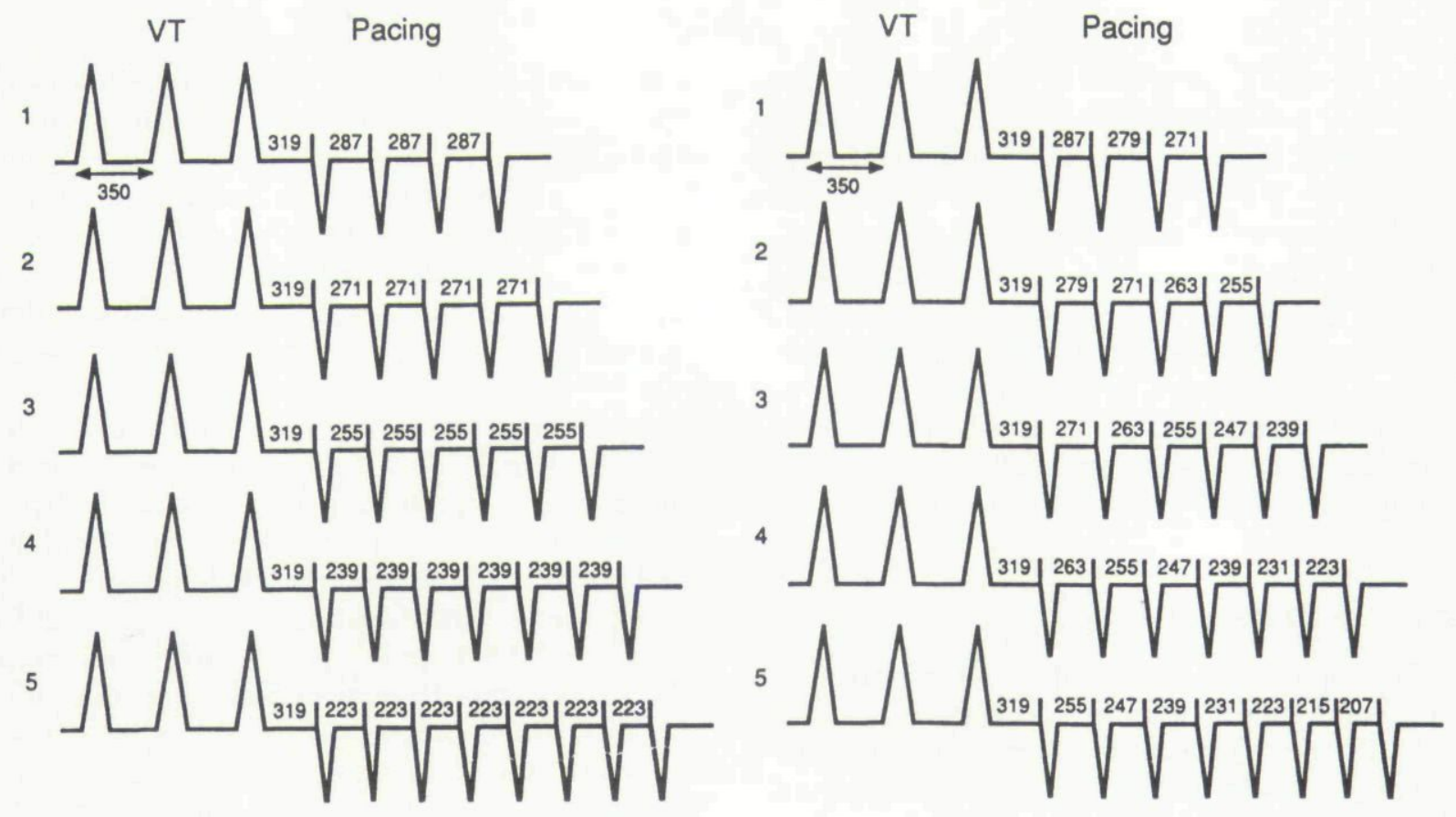

Figure 1. Schematic diagram of the fixed burst (left) and decremental burst (right) pacing algorithms used in this study. The intervals shown are in msec based on a ventricular tachycardia (VT) cycle length of $350 \mathrm{msec}$. The initial stimulus of the pacing train is introduced at a coupling interval of $91 \%$ of the VT cycle length and the second stimulus of the train is delivered at a coupling interval of $81 \%$ of the VT cycle length. A maximum of five attempts are delivered.

at a coupling interval of $81 \%$ of the VT cycle length. Each subsequent impulse was delivered at a coupling interval $8 \mathrm{msec}$ shorter than the previous coupling interval. The initial number of pulses delivered was four. During each successive attempt, the number of pacing impulses was increased by one to a maximum of eight stimuli. The minimal coupling interval used for fixed burst and decremental burst pacing was $200 \mathrm{msec}$. The pacing algorithm used for decremental burst pacing was chosen because a prior study of decremental burst pacing by Charos et al. ${ }^{4}$ reported that $80 \%$ of episodes of VT were terminated with a 7- to 8-beat train and that the optimal beat-by-beat decrement was $8-10$ msec. To allow an accurate comparison of fixed burst and decremental burst pacing, the fixed burst pacing algorithm was identical to the decremental burst pacing algorithm with the exception of a slightly larger decrement between successive trains of stimuli.

\section{Statistical Analysis}

All data are presented as mean \pm 1 standard deviation. The data were compared using Stu- dent's t-tests and Chi-square analysis. In all cases a $\mathrm{P}<0.05$ was considered significant.

\section{Results}

\section{Efficacy of Fixed Burst Pacing}

The efficacy of fixed burst pacing is summarized in Table I. Forty of $57(70 \%)$ episodes of VT were successfully terminated, $21 \%$ of VT episodes were accelerated, and $9 \%$ of VT episodes were unchanged. The effectiveness of fixed burst pacing was greater for VTs with a cycle length of $300 \mathrm{msec}$ or more than for VTs with shorter cycle lengths ( $76 \%$ vs $45 \%, \mathrm{P}=0.04$ ). The risk of VT acceleration was also related to the VT rate, being greater for VTs with a cycle length $<300 \mathrm{msec}$ than for VTs with longer cycle lengths $(55 \%$ vs $13 \%$, P < 0.001 ). In each case of acceleration, ventricular fibrillation or polymorphic VT was induced and required cardioversion to terminate. The efficacy of fixed burst pacing was no different for VTs of different morphologies $(P>0.1)$. A histogram of the number of stimuli required for VT termination is 


\section{Table I.}

Efficacy of Fixed Burst Pacing

\begin{tabular}{lcrrl}
\hline & Number of VT Episodes & Success & Acceleration & Failure \\
\hline All VTs & 57 & $40(70 \%)$ & $12(21 \%)$ & $5(9 \%)$ \\
VT CL $>300 \mathrm{msec}$ & 46 & $35(76 \%)$ & $6(13 \%)$ & $5(11 \%)$ \\
VT CL $<300 \mathrm{msec}$ & 11 & $5(45 \%)$ & $6(55 \%)$ & $0(0 \%)$ \\
\hline
\end{tabular}

$\mathrm{CL}=$ cycle length; $\mathrm{VT}=$ ventricular tachycardia.

shown in Figure 2. When successful, a mean of 511 stimuli of fixed burst pacing were required for VT termination (mean number of trains $=211$ ).

\section{Efficacy of Decremental Burst Pacing}

The efficacy of decremental burst pacing is summarized in Table II. Forty-one of $57(72 \%)$ of VT episodes were successfully terminated, $18 \%$ of VT episodes were accelerated, and $10 \%$ of VT episodes were unchanged. The effectiveness of decremental burst pacing was greater for VTs with a cycle length of at least $300 \mathrm{msec}$ than for VTs with shorter cycle lengths $(80 \%$ vs $36 \%$, P < 0.001 ). The risk of VT acceleration was also related to the VT rate, being greater for VTs with a cycle length $<300 \mathrm{msec}$ than for VTs with longer cycle lengths ( $55 \%$ vs $9 \%, \mathrm{P}<0.001)$. In each case of acceleration, ventricular fibrillation or polymorphic VT was induced and required cardioversion to terminate. The efficacy of decremental burst pacing was no different for VTs of different mor-

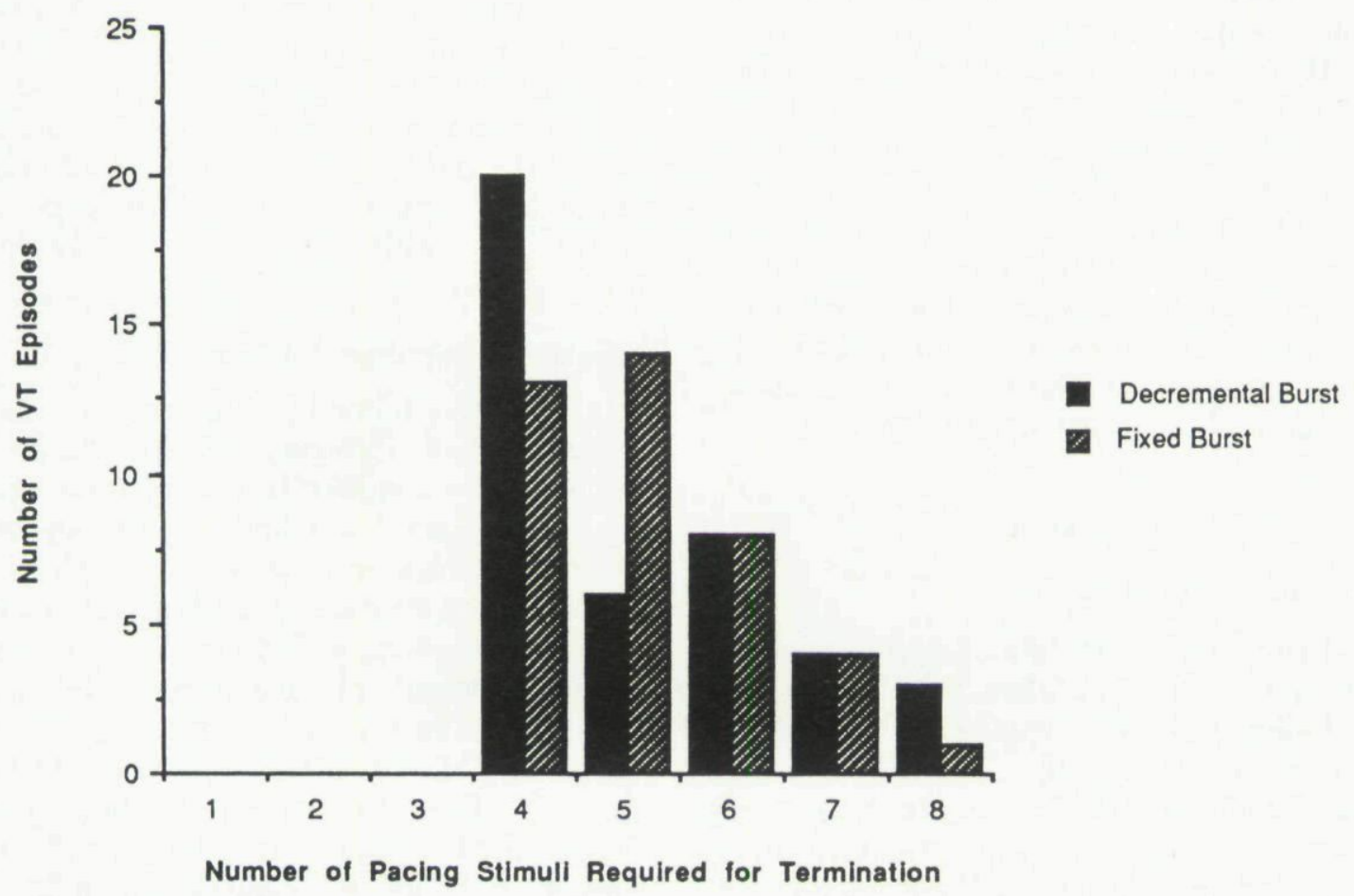

Figure 2. The distribution of the number of pacing stimuli required for termination of ventricular tachycardia (VT) with decremental burst and fixed burst pacing is shown. The number of VT episodes that were successfully terminated by each pacing algorithm is shown on the vertical axis and the number of stimuli in the successful pacing train is shown on the horizontal axis. 
Table II.

Efficacy of Decremental Burst Pacing

\begin{tabular}{lcccc}
\hline & Number of VT Episodes & Success & Acceleration & Failure \\
\hline All VTs & 57 & $41(72 \%)$ & $10(18 \%)$ & $6(10 \%)$ \\
VT $C L>300 \mathrm{msec}$ & 46 & $37(80 \%)$ & $4(9 \%)$ & $5(11 \%)$ \\
VT $C L<300 \mathrm{msec}$ & 11 & $4(36 \%)$ & $6(55 \%)$ & $1(9 \%)$ \\
\hline
\end{tabular}

$\mathrm{CL}=$ cycle length; $\mathrm{VT}=$ ventricular tachycardia.

phologies $(\mathrm{P}>0.1)$. A histogram of the number of trains of pacing stimuli required for VT termination is shown in Figure 2. When successful, a mean of 511 stimuli of decremental burst pacing were required for VT termination (mean number of trains $=211$ ).

\section{Comparative Efficacy of Fixed Burst and Decremental Burst Pacing}

The two pacing algorithms did not differ in efficacy of VT termination or incidence of VT acceleration $(\mathrm{P}>0.1)$. The response to the two pacing algorithms was concordant during attempts at termination of 48 of 57 VTs ( $84 \%$ ). Five VTs were successfully terminated with decremental burst pacing but were not terminated with fixed burst pacing. Four VTs were successfully terminated with fixed burst pacing but were not terminated with decremental burst pacing. The number of trains of burst pacing and distribution of the number of pacing trains required for VT termination were also no different ( $\mathrm{P}>0.1$, Fig. 2).

\section{Discussion}

\section{Efficacy of Fixed Burst Pacing}

Fixed burst pacing terminated approximately $70 \%$ of episodes of VT. The efficacy of VT termination was higher and the incidence of acceleration of VT was lower for VTs with a cycle length $>300$ msec than for more rapid VTs. No previous study has reported the efficacy of fixed burst pacing delivered in a precise automated fashion using an increasing number of stimuli with each successive attempt. However, the findings of this study are consistent with those of prior studies that have examined the efficacy of fixed burst pacing deliv- ered during electrophysiological testing or in the coronary care unit. ${ }^{4-8}$ Roy et al. ${ }^{5}$ reported successful termination of $76 \%$ of VT episodes with fixed burst pacing. The efficacy of VT termination decreased to $40 \%$ for VTs with a cycle length shorter than 300 msec. Naccarelli et al. ${ }^{6}$ reported a similar efficacy of fixed burst pacing (61\%). The presence of a relationship between VT cycle length and the ease of termination VT termination was also observed with $76 \%$ of VTs with a cycle length $>350$ msec terminated by fixed burst pacing versus 51\% of VTs with a shorter cycle length. Charos et al. ${ }^{4}$ reported a somewhat lower efficacy of VT termination with fixed burst pacing (56\%) whereas Fisher et al. ${ }^{7}$ reported a higher efficacy of VT termination $(89 \%)$. These differences may reflect differences in the patient population studied, the cycle lengths of the VTs, as well as the precise pacing algorithm that was used.

\section{Efficacy of Decremental Burst Pacing}

Decremental burst pacing terminated approximately $70 \%$ of episodes of VT. The efficacy of VT termination was higher and the incidence of acceleration of VT was lower for VTs with a cycle length $>300 \mathrm{msec}$ than for more rapid VTs. These results are consistent with those of Charos et al. ${ }^{4}$ who reported a $92 \%$ efficacy of VT termination (VT cycle length $>280 \mathrm{msec}$ ) with a decremental burst pacing protocol very similar to the one used in this study. The higher efficacy reported by Charos et al. ${ }^{4}$ may reflect differences in the patient population studied and the cycle lengths of the VTs. Charos et al. ${ }^{4}$ had previously demonstrated that the efficacy of VT termination was greater $(78 \%$ vs $43 \%$ ) and the incidence of VT acceleration smaller ( $2 \%$ vs $17 \%$ ) for VTs with a cycle length longer than $280 \mathrm{msec}$ than for more rapid VTs. 


\section{Efficacy of Fixed Burst versus Decremental Burst Pacing}

The results of this study demonstrate that fixed burst and decremental burst pacing are equally effective in terminating VT and result in a similar incidence of VT acceleration. These findings are in contrast to those of Charos et al., ${ }^{4}$ who reported that decremental burst pacing was more effective in terminating VT than was fixed burst pacing ( $92 \%$ vs $56 \%, \mathrm{P}<0.001)$. This difference may reflect differences in the pacing algorithms used. In the present study the decremental burst and fixed burst pacing algorithms that were tested differed only in whether the coupling interval between successive pacing stimuli was fixed (fixed burst) or decreased (decremental burst). In contrast, the two pacing algorithms tested by Charos et al. ${ }^{4}$ also differed in many ways. These differences included the number of extrastimuli, the coupling intervals, and whether the trains were triggered manually or in an automated fashion.

\section{Limitations}

There are several limitations to this study. First, this study evaluated the efficacy of two antitachycardia pacing algorithms. Therefore, the results of this study may not be applicable to other fixed burst and decremental burst pacing algorithms that use different numbers of extrastimuli, different coupling intervals, and different coupling interval decrements. And second, the majority of patients in this study were being treated with antiarrhythmic drug therapy that may make VT either easier or more difficult to terminate ${ }^{5,6}$ However, it is very unlikely that antiarrhythmic drug therapy would have altered the relative ability of burst pacing versus decremental pacing to terminate VT. Furthermore, in this study the efficacy of antitachycardia pacing in terminating VT (cycle length $>300 \mathrm{msec}$ ) was no different for VTs in-

\section{References}

1. Herre JM, Cannom DS, Fogoros RN, et al. Initial multicenter clinical experience with a multifunctional implantable pacemaker defibrillator. Circulation 1991; 84:II-428.

2. Ellenbogen K, Luceri R, Dorian P, et al. Clinical evaluation of implantable cardioverter defibrillator duced in the presence or absence of antiarrhythmic drug therapy ( $74 \%$ vs $80 \%$, respectively, $\mathrm{P}>$ $0.2)$.

\section{Clinical Implications}

Presently, several implantable cardioverter defibrillators that are capable of antitachycardia pacing as well as cardioversion/defibrillation are in clinical trials. ${ }^{1-3}$ The devices are programmable and are capable of delivering an extremely large number of different pacing algorithms. Based on the results of this and previous studies, several recommendations can be made that may minimize the number of pacing algorithms that are tested when programming these devices. First, antitachycardia pacing should generally not be used for termination of VTs with a cycle length of $<300 \mathrm{msec}$, because the efficacy of antitachycardia pacing is extremely low and the risk of VT acceleration is high. Second, an adaptive pacing scheme similar to the one used in this study is effective in terminating approximately $75 \%$ to $80 \%$ of VTs that have a cycle length $>300 \mathrm{msec}$. Therefore, in patients with multiple types of VT, a single adaptive pacing scheme may be effective for all VTs. Third, a mean of five pacing stimuli are required for VT termination and only rarely does VT termination require eight stimuli. Therefore, initial testing of pacing algorithms with train lengths of between four and eight stimuli can be recommended. And fourth, although the overall efficacy of fixed burst and decremental burst pacing are no different when the initial pacing algorithm is unsuccessful in terminating VT, there is approximately a $15 \%$ likelihood that the alternate pacing algorithm will be effective. Further studies will be required to determine the relative benefit of testing an alternate pacing algorithm (decremental burst versus fixed burst) versus testing the same pacing algorithm with shorter coupling intervals and/or additional stimuli.
(ICD) function: Importance of data logging to assess device efficacy. Circulation 1991; 84:II-426.

3. Rankin AC, Zaim S, Powell A, et al. Efficacy and limitation of tiered therapy for the treatment of recurrent ventricular arrhythmias. Circulation 1991; 84:II-427. 
4. Charos GS, Haffajee CI, Gold RL, et al. A theoretically and practically more effective method for interruption of ventricular tachycardia: Self-adapting autodecremental overdrive pacing. Circulation 1986; 73:309-315.

5. Roy D, Waxman HL, Buxton AE, et al. Termination of ventricular tachycardia: Role of tachycardia cycle length. Am J Cardiol 1982; 50:1346-1350.

6. Naccarelli GV, Zipes DP, Rahilly T, et al. Influence of tachycardia cycle length and antiarrhythmic drugs on pacing termination and acceleration of ventricular tachycardia. Am Heart J 1983; 1:1-5.

7. Fisher JD, Mehra R, Furman S. Termination of ventricular tachycardia with bursts of rapid ventricular pacing. Am J Cardiol 1978; 41:94-101.

8. Fisher JD, Kim SG, Matos JA, et al. Comparative effectiveness of pacing techniques for termination of well-tolerated sustained ventricular tachycardia. PACE 1983; 6:915-922. 
This document is a scanned copy of a printed document. No warranty is given about the accuracy of the copy. Users should refer to the original published version of the material. 